\title{
Type 2 Autoimmune Hepatitis Due to Acute Epstein Barr Virus Infection or is it the Other Way Around?
}

\author{
Kafayat A Busari* and Juan Ricardo \\ Department of Medicine, Florida State University College of Medicine, USA
}

*Corresponding author: Kafayat A Busari, Department of Medicine, Florida State University College of Medicine, Sarasota, USA.

\begin{abstract}
Introduction
We present a case of a 19-year-old female who presented with abdominal pain and severe pruritus. Viral studies including complete hepatitis panel were negative for acute infection, however acute infectious mononucleosis heterophiles antibodies were positive along with liver kidney microsome (LKM-1) antibody. Patient's liver on MRCP was markedly heterogenous and nodular with areas of confluent hepatic fibrosis consistent with cirrhosis. Subsequent liver biopsy confirmed findings of acute hepatitis with extensive parenchymal extinction and necrosis with moderate to severe mixed inflammation. The patient was initiated on cholestyramine $4 \mathrm{gm}$ pack daily, prednisone and transferred to a tertiary center for liver transplant evaluation.
\end{abstract}

\section{Introduction}

Auto-immune hepatitis (AIH) Type 2 accounts for less than 5\% of known cases in North America and those affected belong either to 2 demographics: those less than age 14 or women. Untreated AIH can progress to cirrhosis and ultimately liver failure, requiring need for liver transplantation. Acute viral infections such as Epstein Barr Virus (EBV) can mimic AIH and has been loosely associated with serving as a catalyst for developing type $1 \mathrm{AIH}$. However, to date, there has been only 1 reported case of type 2 AIH with EBV positive serology in 26- month-old patient [1]. Additionally, to our knowledge no cases reporting an acute EBV infection in the setting of underlying type $2 \mathrm{AIH}$. Which raises the question on if EBV is a catalyst to AIH or if.

\section{Case Report}

19-year-old female with no past medical history presents with three weeks of colicky abdominal pain lasting minutes and occurring once to twice a day. She stated as her abdominal pain increased in duration and frequency, she began to develop lower extremity edema and full body pruritus unrelieved with over the counter emollients or creams. She presented to us for evaluation citing concerns due to a family history involving what she described as "liver issues" on her father's side which uncovered a grandmother with nonalcoholic fatty liver disease (NAFLD). Physical examination showed an uncomfortable appearing young female with mild scleral icterus and jaundice. Excoriations were noted on the patient's neck, behind her ears and forearms bilaterally. Abdominal exam elicited pain on palpation localized to the RUQ and RLQ quadrants with no rebound or guarding.

Initial liver profile resulted with phosphatase 362, aspartate aminotransferases 1171, alanine aminotransferases 604, total bilirubin 17.4, and direct bilirubin 13.4. EBV early antigen (EA) IgG, viral capsid antigen (VCA) IgG, VCA IgM and EBV (nuclear antigen) NA 1 IgG were all positive consistent with EBV infection. LKM-1 antibody was positive. Anti-Hepatitis A, anti-hepatitis B, 
anti-hepatitis C antibodies HIV and cytomegalovirus were negative. Antibodies to anti-smooth muscle (SMA), anti-mitochondrial (AMA), and alpha-1-anti-trypsin were also negative. The patients acetaminophen, ceruloplasmin, alpha fetoprotein markers were normal

MRCP showed a markedly heterogenous and nodular liver with multifocal areas of confluent hepatic fibrosis, trace peri-hepatic ascites and hypertrophy of the lateral segment of the left hepatic lobe. Multiple prominent reactive mesenteric and retroperitoneal lymph nodes were also identified. Transjugular liver biopsy demonstrated acute hepatitis with extensive parenchymal extinction with a single nodule of residual viable hepatocytes surrounded by fibrosis and necrosis with moderate to severe mixed inflammation. The patient was started on IV Lasix for lower extremity edema, cholestyramine $4 \mathrm{gm} /$ daily and $60 \mathrm{mg}$ prednisone/ day for 5 days with minimal improvement in symptoms. She was subsequently transferred to nearby tertiary facility for evaluation of liver transplant. However due to her young age lending to a more favorable prognosis, liver transplant was deemed not clinically warranted. The patient's pruritus and abdominal pain improved.

\section{Discussion}

To our knowledge, there are no case reports of adolescents being diagnosed with AIH type 2 in the setting of an acute EBV infection. Upon literature review, one case linking EBV to type $2 \mathrm{AIH}$ was published in 2013 in a 26 month old patient [1]. Most cases published describe a suggestive relationship of AIH type 1 after EBV exposure. In these rare reports, patients initially presented with either an acute or chronic EBV infection and labs negative for anti-SMA, AMA, LKM-1 or LC antibodies [2-3]. Several months to years later, these patients were found to have an elevation of transaminases and subsequently AIH diagnosed with liver histology and positive anti- SMA, ANA or AMA titers [3, 5-7]. In our case, our young patient was in the convalescent phase of EBV infection when diagnosed with AIH type 2. Prior to hospitalization, she never had symptoms suggestive of AIH or EBV. It would not be impractical to suggest EBV unmasked the patients underlying type $2 \mathrm{AIH}$, but we also believe the patients type $2 \mathrm{AIH}$ may have increased her risk of developing a more robust EBV infection.

One theory is that infections are more likely to occur in those with underlying autoimmune diseases. EBV, the cause of infectious mononucleosis is an infection that commonly affects $90 \%$ of adolescents. So, it raises the question on if a cause -and -effect relationship does indeed exist between EBV and AIH given how common EBV is amongst this particular patient population [4]. To expand further, one would expect more cases of AIH but this is the first case reporting an adolescent with an acute EBV infection and type 2 AIH diagnosis at the same time. An additional theory states that as we age our immune systems lose resiliency. Therefore, a rise in cases of type 2 or type 1 would be expected in those within the general population approaching their 60s'or 70's. Yet, type 2 is universally considered an autoimmune hepatitis found in childhood. There has not been an exact mechanism detailing the pathogenic process of EBV induced AIH. We believe this is largely in part due to the lack of date, thus possibly highlighting the need for large scale research studies delving deeper into this topic. Nonetheless, strong evidence is lacking in linking EBV as a direct cause of AIH.

\section{Acknowledgement}

None.

\section{Conflict of Interest}

No conflict of interest.

\section{References}

1. Zellos A, Spoulou V, Roma-Giannikou E, Karentzou O, Dalekos GN, et al. (2013) Autoimmune hepatitis type-2 and Epstein-Barr virus infection in a toddler: art of facts or an artifact. Ann Hepatol 12(1): 147-151.

2. Shaukat A, Tsai HT, Rutherford R, Anania FA (2005) Epstein-Barr virus induced hepatitis: An important cause of cholestasis. Hepatol Res 33: $24-26$

3. Niller HH, Wolf H, Minarovits J (2008) Regulation and dysregulation of Epstein-Barr virus latency: implications for the development of autoimmune diseases. Autoimmunity 41: 298-328.

4. Vento S, Cainelli F (2004) Is there a role for viruses in triggering autoimmune hepatitis. Autoimmunity Rev 3: 61-69.

5. Rigopoulou EI, Smyk DS, Matthews CE, Billinis C, Burroughs AK, et al. (2012) Epstein- barr virus as a trigger of autoimmune liver diseases. Adv Virol 2012: 987471.

6. Aceti A, Mura MS, Babudieri S, Bacciu SA (1995) A young woman with hepatitis after a sore throat. Lancet 346: 1603.

7. Cabibi D (2008) Autoimmune hepatitis following Epstein-Barr virus infection. BMJ Case Rep 2008: bcr0620080071. 